\title{
El alcohol como problema de salud pública. La responsabilidad de los poderes públicos
}

\author{
Pérez, B.
}

Director General de Drogodependencias. Generalitat Valenciana.

Enviar correspondencia: Bartolomé Pérez Gálvez. Dirección General de Drogodependencias. Generalitat Valenciana. Alameda, 16. 46010 Valencia. E-mail: bartolomé.perez@sanidad.m400.gva.es

\section{RESUMEN}

El alcohol es la causa del mayor número de casos de adicción a sustancias, si exceptuamos la producida por el tabaco. De hecho, un 9.5\% de la población española entre 15 y 65 años presenta patrones de consumo abusivo y se estima que un $2 \%$ de esta población podría cumplir criterios diagnósticos de dependencia alcohol. La intensidad del problema generado por el consumo abusivo de alcohol, junto a estos datos, se manifiesta en la relación de causalidad con el 3.4\% de la mortalidad anual. En términos económicos, el abuso de alcohol produce unos costes anuales que, en España, llegan a superar anualmente los 800.000 millones de pesetas.

Ante esta situación, las distintas Administraciones Públicas están obligadas a planificar y desarrollar políticas globales. Estas políticas deben englobar acciones dirigidas a tanto a disminuir la accesibilidad a las bebidas alcohólicas como a incrementar la información de la sociedad y limitar el efecto de la publicidad en el individuo. Estas acciones deben complementarse con otras dirigidas a reducir el daño asociado al consumo de alcohol en determinadas situaciones con la oferta de una asistencia normalizada y basada en la evidencia científica.

Palabras clave: Política, alcohol, alcoholismo, salud pública, administración.

\begin{abstract}
Alcohol is the cause of most of the substance abuse cases, excluding tobacco. In fact, $9.5 \%$ of Spanish population aged 15 to 65 are risky drinkers, and it is estimated that $2 \%$ of the population meet criteria for alcohol dependence. The intensity of the problem generated by the abusive alcohol consumption, near these data, is manifested in the relationship of causality with $3.4 \%$ of the annual mortality. In economic terms, the alcohol abuse produces some annual costs that, in Spain, arrive to surpass annually 800.000 millions of pesetas.

Before this situation, the different Public Administrations are obligated to plan and develop global policies. These policies should include actions directed to reduce the accessibility to the alcoholic beverages as well as to increase the information of the society and to limit the effect of the advertising in the individual. These actions should be complemented with other directed to reduce the damage associated with alcohol consumption in given situations with the offer of a normalised assistance and based on the scientific evidence.
\end{abstract}

Key words: Alcohol Policies, alcoholism, public health, public administration. xceptuando el tabaquismo, la dependencia al alcohol se sitúa como la primera patología adictiva en nuestro país. Un 9.5\% de la población española entre 15 y 65 años consumen alcohol de forma abusiva ${ }^{1} y$, cuando menos, un $2 \%$ de la población general podría cumplir criterios de Dependencia al Alcohol $^{2}$. Entre 1997 y 1999, el número de 
ingresos hospitalarios por Síndrome de Dependencia al Alcohol se ha incrementado un $35 \%$, situando la tasa española en 21.02 ingresos por cada 100.000 habitantes.

Con relación al consumo per cápita de alcohol, cabe reseñar que, entre 1980 y 1998, se ha producido un descenso del $25 \%$, relacionado fundamentalmente con la caída en el consumo de vino ${ }^{3}$. Sin embargo, y como posteriormente expondremos, la tendencia en los dos últimos años, con relación a las compras de vinos, cervezas y licores por la población española, es claramente alcista.

Estos datos definen claramente la intensidad de un problema que, solo muy recientemente y con la aprobación de la Estrategia Nacional sobre Drogas 2000-20084, ha sido incluido dentro del contexto de las drogodependencias en nuestro país.

El presente capítulo no pretende alcanzar un nivel de profundización exhaustivo en aquello que ha venido denominándose "alcohol policy". Por el contrario, su objetivo es incidir en la importancia de la responsabilidad de los poderes públicos en uno de los principales problemas de salud de la población española, responsabilidad que debe generarse como respuesta ante la magnitud del problema.

Con este fin, expondremos un breve recuerdo sobre los problemas que, en términos de costes, morbilidad y mortalidad, genera el alcohol en la sociedad. No deben olvidarse los beneficios que, cuando menos en términos económicos, genera uno de las principales sectores productivos de nuestro país. Finalmente, y en un intento por alcanzar un óptimo balance entre ambos extremos, se proponen distintas acciones, de contrastada utilidad, como elementos constitutivos de lo que entendemos como una adecuada, y necesaria, política global ante el abuso de alcohol por parte de la población española.

\section{LOS COSTES SOCIALES Y SANITARIOS DEL ABUSO DE ALCOHOL}

Los estudios de costes son objeto de otro capítulo en esta misma obra. No obstante, entendemos que no es posible alcanzar una visión global de las políticas públicas en esta materia sin, previamente, hacer una breve mención al daño generado a la sociedad, en términos económicos, por el abuso de alcohol.

Este tipo de estudios analizan la repercusión económica que genera el abuso de alcohol, generalmente valorando tres áreas básicas de una población determinada:

a) La atención sanitaria, incluyendo tanto la morbilidad como la mortalidad atribuibles al abuso de alcohol.

b) El bienestar social, con referencia a factores de tipo laboral y a las distintas prestaciones sociales.

c) La seguridad ciudadana, en especial respecto a los accidentes y a la delincuencia asociada.

Edwards y colaboradores ${ }^{5}$ realizan ciertas observaciones que deben ser consideradas. Por una parte, advierten sobre la inexactitud de la mayoría de los estudios, especialmente con referencia a los costes atribuidos al desempleo. Por otra, consideran que los dramáticos resultados que genera el abuso de alcohol en la sociedad, invalida cualquier intento por hallar un equilibrio coste-beneficio en términos exclusivamente económicos.

Muy escasos han sido los estudios realizados en nuestro país, relativos al coste producido por el abuso de alcohol. En su mayoría, estos estudios se centran en la valoración de los costes sanitarios, constituyendo una primera aproximación al coste global. Así, entre los años 1993 y 1994 los gastos hospitalarios generados por el abuso de alcohol en la Comunidad Valenciana se estimaban en cerca de 12.000 millones de pesetas. En otros términos, un $5 \%$ del gasto total hospitalario en el mismo periodo, siempre según los cálculos más optimistas.

En la escasa obra existente, destaca sobremanera el análisis realizado por Portella y colaboradores $^{2}$. En este caso, los autores analizaron los costes atribuibles al abuso de alcohol en España, siguiendo el esquema expuesto en la Tabla I. 


\title{
Tabla I.-Tipos de costes atribuibles al abuso de alcohol (Portella et al., 1998).
}

\author{
Costes directos \\ - Utilización de servicios sanitarios. \\ - Pérdidas materiales derivadas de accidentes de tráfico. \\ - Actuaciones de la Administración derivadas de la actividad criminal. \\ - Otros costes. \\ Costes indirectos \\ - No sanitarios (subsidios, pérdidas de productividad). \\ - Sanitarios. \\ Costes intangibles
}

Según este análisis, el gasto anual generado por los problemas relacionados con el abuso de alcohol, en nuestro país, podría ascender a 637.717 millones de pesetas (año 1997). De ellos, un 35.82\% corresponderían a costes directos y el restante $64.18 \%$ a costes indirectos.

Los propios autores establecen ciertas consideraciones respecto a las limitaciones de su estudio. Entre ellas destaca el hecho de que, por distintos motivos, no se hubiesen computado gastos de especial interés como los generados por consultas externas hospitalarias y de especialidades ambulatorias, las indemnizaciones por muerte o invalidez causadas por accidentes, el Síndrome Alcohólico Fetal así como los años potenciales de vida y de vida productiva perdidos (APVP). Esta última variable (APVP) alcanza un total de 224.370 años. No obstante, los autores optaron por no contabilizarla en términos económicos, ante la imposibilidad de atribuir un coste determinado a un año de vida, así como a sus posibles diferencias según grupos sociales.

Por otra parte, Portella y colaboradores advierten que los costes directos sobre la atención sanitaria deben ser entendidos como un problema de coste-oportunidad. Esto es, la disminución de los problemas relacionados con el abuso de alcohol no generaría realmente un ahorro económico, sino la posibilidad de destinar estos recursos a otros fines sanitarios. Por el contrario, los costes que repercuten en el mundo laboral sí pueden producir un ahorro económico real, tanto a las empresas como a las entidades aseguradoras.

Con relación al Producto Interior Bruto, y siempre según los datos del citado estudio, las consecuencias derivadas del abuso de alcohol podrían significar el $0.8 \%$ del PIB de nuestro país. Actualizando este resultado al PIB del año 2000, y aceptando las limitaciones expuestas en el estudio de Portella y colaboradores, el gasto generado por el abuso de alcohol superaría los 800.000 millones de pesetas en ese año.

Otros estudios ${ }^{6}$ llegan a situar este porcentaje en el $1.6 \%$ de la renta per cápita norteamericana. En Canadá', los costes derivados del abuso de alcohol se estiman en el 1.09\% del PIB canadiense. En el estudio canadiense, el costo atribuible a drogas ilegales apenas alcanzaba el $0.2 \%$ del PIB. Solo el coste generado por el tabaquismo (1.39\% PIB) superaba al motivado por el abuso de alcohol.

No parece existir homogeneidad a la hora de estimar los costes atribuibles al abuso de alcohol. Razones metodológicas suelen justificar discrepancias entre estos datos y otros que, como los obtenidos en el estudio realizado por Eurocare en 1995, llegan a situar el coste atribuible al abuso de alcohol en un 5$6 \%$ del PIB europeo. A este respecto, debe tenerse en cuenta el hecho de que, siendo el consumo medio de alcohol en los países europeos, sensiblemente más elevado que en Estados Unidos o Canadá, es lógico espe- 
rar un coste económico proporcionalmente más elevado.

\section{MORBILIDADY MORTALIDAD ASOCIADAS AL ABUSO DE ALCOHOL}

\section{Según el Global Status Report on Alcohol} publicado por la Organización Mundial de la Salud $^{8}$, el abuso de alcohol motiva el 1.5\% de la mortalidad mundial cada año. Esta cifra se elevaba considerablemente en los países desarrollados, donde la malnutrición y las carencias higiénico-sanitarias (principales causas de muerte a nivel mundial) apenas están representadas. En este tipo de países, la propia OMS estima que el alcohol es responsable del $11 \%$ de las enfermedades y muertes acontecidas cada año ${ }^{9}$.

El consumo inadecuado de alcohol es responsable, igualmente, del $2.1 \%$ de los años potenciales de vida perdidos. Por otra parte, su capacidad para afectar crónicamente a la persona favorece que se relacione directamente con el $6 \%$ de los años de vida con discapacidad y el $3.5 \%$ del total de años de vida ajustados por discapacidad. En este sentido, el abuso de alcohol se sitúa como la octava causa de mortalidad y el primer factor generador de años vividos con discapacidad ${ }^{\text {. }}$.

El conocimiento de la morbilidad y mortalidad asociadas al abuso de alcohol constituye una útil herramienta para valorar:

a) La intensidad del problema en términos de pérdida de salud de la población.

b) La prevalencia indirecta del abuso de alcohol, conjuntamente con otros indicadores.

Previamente a realizar una breve descripción de la mortalidad y morbilidad asociadas al abuso de alcohol, tal vez sea conveniente recordar la influencia del tipo de consumo sobre la evolución de aquellas. Determinadas patologías y causas de muerte ofrecen una mayor relación con un consumo de tipo agudo o "explosivo", como los accidentes, las intoxicaciones o los actos violentos ${ }^{2}$. Otras, como la cirrosis o las neoplasias, orientarán más hacia el componente "crónico" del patrón de consumo de una población o sociedad concreta. Esta simple delimitación debe ser considerada, con posterioridad, en el momento de establecer políticas concretas de intervención. Por ello, el cómputo global de morbilidad y mortalidad asociadas al abuso de alcohol ofrece importantes limitaciones, si exceptuamos su valor como indicador de la magnitud general del problema. Este cálculo debe diferenciar claramente las patologías o causas de muerte, al objeto de analizar su evolución individual.

Es preciso considerar que ciertas patologías, como algunas de las neoplásicas o las cardiovasculares, presentan múltiples factores de riesgo distintos al consumo de alcohol, que pueden modificar sensiblemente las prevalencias de estas patologías. Además, aun cuando la fracción atribuible al consumo de alcohol fuese elevada, en ocasiones puede tratarse de patologías con bajas prevalencias, circunstancia ésta que dificulta su seguimiento a lo largo del tiempo.

Por estos motivos, Edwards ${ }^{2}$ aconseja analizar la evolución de la mortalidad o la morbilidad asociada cuando la fracción atribuible al consumo de alcohol sea elevada. En sintonía con este planteamiento, la Organización Mundial de la Salud ${ }^{8}$ utiliza como indicadores las tasas de mortalidad correspondientes a cirrosis y otras enfermedades crónicas hepáticas ( $F A=70 \%)$ y a accidentes de tráfico de vehículos de motor ( $F A=40 \%)$. Como anteriormente comentábamos, estos indicadores permiten discriminar dos patrones de consumo diferenciados: uno más crónico, asociado a las patologías hepáticas, y otro más agudo, relacionado con los accidentes de tráfico.

Con relación a la morbilidad asociada, destacan los estudios realizados por distintos autores, en los que se determinan la fracción atribuible al alcohol de distintas patologías, en Estados Unidos, Australia y Canadá, respectivamente. Por su interés, aconsejamos la lectura de una interesante exposición de los hallazgos de estas investigaciones, que 
puede ser consultada en una reciente publicación de la $\mathrm{OMS}^{10}$.

Nos centraremos, pues, en un análisis algo más detallado de la mortalidad relacionada con el abuso de alcohol. Para ello, es común la utilización de las fracciones atribuibles propuestas por los Centres for Disease Control (CDC) de Estados Unidos, expuestas en la Tabla II.

Tabla II. Mortalidad atribuible al alcohol.

\section{Causa de fallecimiento}

Cirrosis y otras enfermedades crónicas del hígado

Accidentes de tráfico de vehículos a motor

Pancreatitis aguda

Psicosis alcohólica

Síndrome de Dependencia Alcohólica

Abuso de Alcohol

Envenenamiento accidental por alcohol, no clasificados en otra parte

Suicidios y lesiones autoinfligidas

Cardiomiopatía alcohólica

Gastritis alcohólica

Polineuropatía alcohólica

Pancreatitis crónica

T. Maligno labio, cavidad oral, faringe

T. Maligno esófago

T. Maligno laringe

Homicidio
Fracción atribuible

$70 \%$

$40 \%$

$42 \%$

$100 \%$

$100 \%$

$100 \%$

$100 \%$

$25 \%$

$100 \%$

$100 \%$

$100 \%$

$60 \%$

$50 \%$

$75 \%$

$50 \%$

$46 \%$
Según los últimos datos disponibles en nuestro país ${ }^{1}$, el alcohol sería responsable de 12.032 fallecimientos en el año 1999, cifra que representa un $3.44 \%$ de todas las defunciones registradas en ese año.

Debe advertirse que, al igual que en la determinación de costes, existe cierta falta de homogeneidad en la determinación del número de muertes relacionadas con el alcohol. Especialmente llamativa es la confusión que, tal vez con objeto de llamar la atención sobre la gravedad del problema, genera la propia OMS. Baste observar cómo, en el Plan Europeo de Actuación sobre Alcohol 20002005 , se le atribuye a éste entre el $40 \%$ y el $60 \%$ de las muertes de la Región Europea.

Evidentemente, las fracciones atribuibles pueden variar sustancialmente entre los estudios americanos y los europeos. Existen, por otra parte, grandes diferencias entre algunos de los países que constituyen la Región Europea de la OMS. Pero, con todo, nos parece una diferencia tan relevante que aconseja la obtención de fracciones atribuibles específicas en cada país. Del mismo modo, y por este motivo, debe valorarse con cierto recelo este tipo de cálculos globales y asumir la citada advertencia de Edwards ${ }^{2}$, al respecto de centrar el análisis de la mortalidad en las patologías con fracción atribuible casi específica del abuso de alcohol.

Al margen de ofrecer una visión global de la incidencia del alcohol en la mortalidad de la población, este indicador puede ser igualmente útil en el seguimiento del consumo medio de una población determinada. Aún más, permite diferenciar las características cualitativas de éste (crónico-regular o agudo-explosivo) y, en consecuencia, es un instrumento útil en el diseño de acciones para disminuir el daño relacionado con el consumo de alcohol.

Como ejemplo de su utilidad, el Gráfico 1 expone la evolución del consumo per cápita de la población española, así como de tres indicadores de mortalidad: 
a) La motivada por cirrosis y otras enfermedades hepáticas crónicas, como indicador relacionado con el consumo regular de alcohol. b) La producida por accidentes de tráfico y por actos violentos (homicidio y suicidio), que mostrarían una relación más directa con el consumo agudo o explosivo.

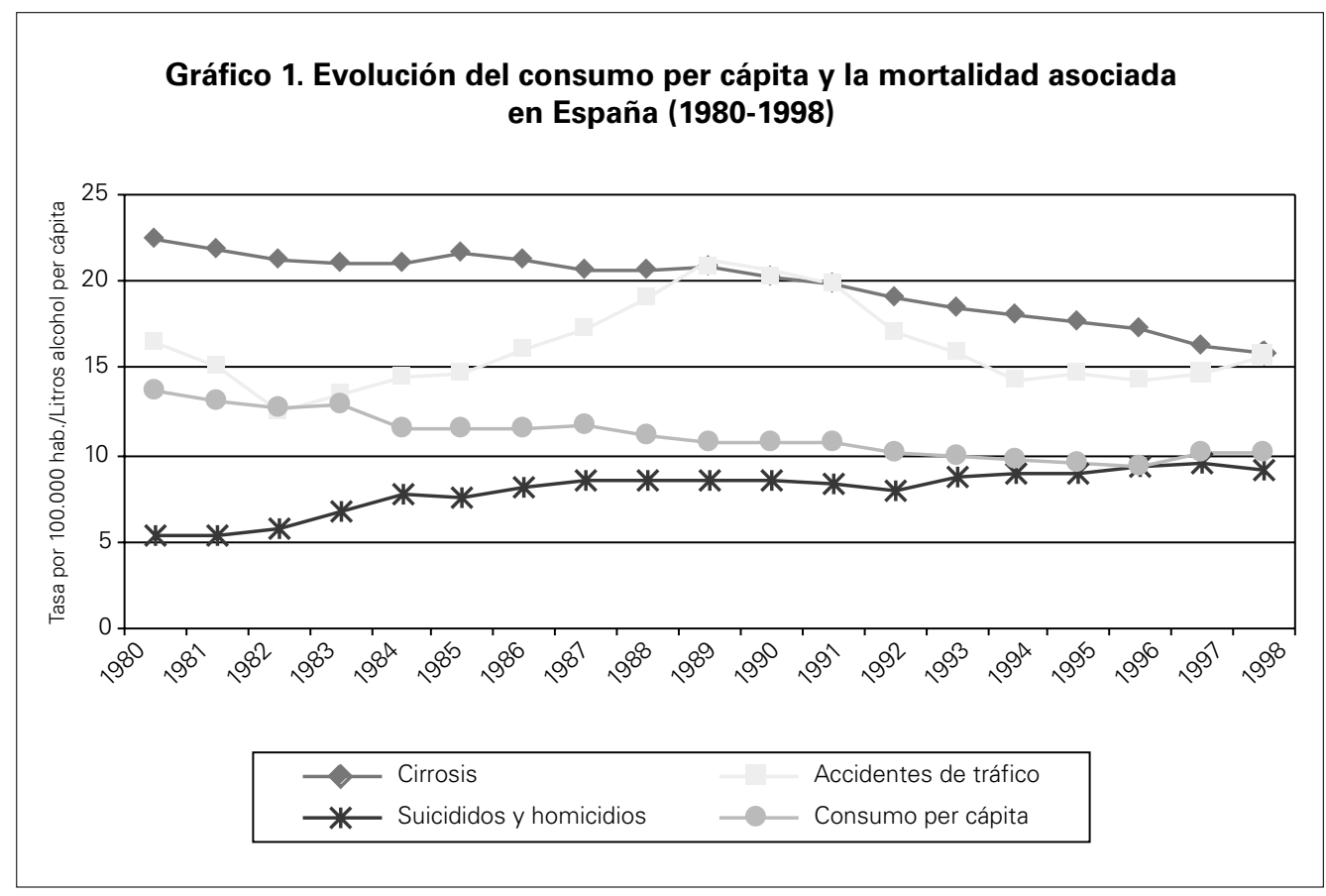

Como se puede observar en el Gráfico 1, solo la mortalidad por cirrosis muestra una relación directa con la evolución del consumo per cápita $(r=.830)$. Por el contrario, la mortalidad por accidentes de tráfico y por actos violentos ofrecen relaciones inversas, en este último caso más relacionada con los suicidios que con los homicidios, donde sí existe una ligera relación directa $(r=.290)$ aunque no significativa.

Los resultados obtenidos nos permiten afirmar que:

a) Aun con sus limitaciones respecto al momento de inicio de la patología, la tasa de mortalidad por cirrosis sigue siendo un buen indicador del abuso de alcohol en la población general española.

b) La mortalidad por accidentes de tráfico y por violencia, están más relacionadas con los aspectos cualitativos del consumo que con los cuantitativos. En otros términos, no se relacionan directamente con el consumo per cápita de una población, sino con el modo de consumo intensivo o abuso agudo de alcohol.

c) En su aplicación práctica, estas diferencias obligan a planificar acciones, complementarias entre si, que disminuyan tanto los efectos generados por el consumo crónico como por el agudo.

Con relación a este último supuesto, cabe reseñar que el daño producido por el abuso regular de alcohol, en términos de mortalidad, sigue siendo sensiblemente superior. Como ejemplo, las muertes por cirrosis en 1997, atribuidas al abuso de alcohol, duplicaron a las producidas por accidente de tráfico igualmente atribuidas al alcohol. Por ello es 
preciso llamar la atención de los gestores públicos al respecto de no polarizar la atención sobre determinados problemas como los accidentes de tráfico, debiéndose mostrar similar interés (cuando no superior) por otros problemas, de mayor magnitud, generados igualmente por el abuso de alcohol.

\section{LA INDUSTRIA DEL ALCOHOL: SU PRO- DUCCIÓN E INGRESOS ECONÓMICOS}

Un factor de especial relevancia en la definición de políticas públicas en materia de consumo y abuso de alcohol es, indudablemente, la trascendencia económica de su producción y comercio. Citábamos anteriormente la observación de Griffith Edwards, al respecto de lo desafortunado de buscar ante este problema una simple relación de coste-beneficio. Efectivamente, las consecuencias del abuso de alcohol no deben ser entendidas, única y exclusivamente, desde una óptica economicista. Pero, por otra parte, tampoco deben obviarse los beneficios económicos que, para un país como España, generan la producción y comercio de bebidas alcohólicas.

La histórica posición de España, entre las grandes potencias productoras de bebidas alcohólicas, sigue consolidándose año tras año. Con 36.080 millones de hectolitros, España es el tercer país productor de vino, posición que desciende hasta el décimo lugar si a la cerveza nos referimos, con una nada despreciable producción de 24.773 millones de hectolitros ${ }^{3}$.

Las bebidas alcohólicas representan el $11.3 \%$ de las compras alimentarias de la población española'. En su manifestación económica, las compras efectuadas en 1999 superan ligeramente el billón de pesetas, esto es, el $1.07 \%$ del PIB español registrado en ese ejercicio. Con un total de 3.809 millones de litros adquiridos en el año 1999 y una ratio per cápita cifrada en 95.3 litros, la evolución desde 1995 ha mantenido una ligera tendencia a la baja que, sin embargo, merece realizar ciertas matizaciones.
Esta evolución ha sido dispar, dependiendo del tipo de bebidas. Hasta 1999, es llamativo el comportamiento inversamente relacionado con la graduación alcohólica. No obstante, esta tendencia parece empezar a quebrarse si comparamos los nueve primeros meses de los años 1999 y 2000 (últimos datos disponibles), con un incremento del $16.89 \%$ en el volumen de licores adquiridos por los españoles. Aunque apenas varíe el volumen total de compras, la mayor representación de los licores hace esperar un posible incremento en el consumo per cápita de alcohol.

La exportación de bebidas alcohólicas ocupa un destacado lugar en el contexto del comercio exterior español. Según los datos registrados por la $\mathrm{FAO}^{8}$, correspondientes al ejercicio anual de 1998, España es el tercer país exportador de vinos a nivel mundial. Cabe reseñar que el volumen de vinos exportado por los tres primeros países (Francia, Italia y España) triplica la suma de las siete naciones que les siguen en el "top ten". Por otra parte, la evolución exportadora española es sensiblemente más favorable que la de sus más directos competidores: entre 1996 y 1998, las exportaciones españolas de vino se incrementaron un $47.14 \%$ frente al $27.40 \%$ registrado en Francia y el $11.56 \%$ de Italia.

En el capítulo de países exportadores de licores, España ya ocupa la décima posición. Sin embargo, esta situación contrasta con ser el tercer país con mayor volumen de importaciones de licores, con un balance sensiblemente favorable a los de producción extranjera. Situación similar se observa con relación a la cerveza, en cuyo caso nuestro país ofrece una producción en su mayoría destinada al comercio interior, reforzada con una importación que la sitúa en el noveno lugar a nivel mundial.

Obviamente, la trascendencia de la balanza comercial exterior se manifiesta en beneficios económicos. Y, si a éstos nos referimos, España ocupa la sexta posición en el ranking de países exportadores de toda clase de bebidas alcohólicas, por ingresos económicos generados. En 1995, nuestro país obtuvo ingresos por valor de 1.141.783.000 US\$ 
como resultado de este tipo de exportaciones. En términos porcentuales, las exportaciones de bebidas alcohólicas significaron, en ese año, el $1.25 \%$ del total de ganancias registradas por el comercio exterior español. Con todo, este porcentaje es sensiblemente inferior al observado en otros países que, como Moldavia (24.03\%) o la Martinica $(10.81 \%)$ generan gran parte de sus ingresos exportadores por medio de la venta de bebidas alcohólicas.

En el extremo opuesto, España también destaca en el ranking mundial de países importadores de bebidas alcohólicas, en cuanto a sus costes se refiere. En el mismo año, nuestro país gastó 1.068.446.000 US\$ en importar bebidas alcohólicas, en su mayoría destiladas. Un gasto que sitúa a España como séptimo país importador y que representa el $0.93 \%$ del total de importaciones por su coste económico.

Todo lo expuesto nos lleva a concluir la importancia de la producción y comercio de las bebidas alcohólicas, dentro del contexto económico nacional. Una realidad que, lejos de obviarse, debe ser tenida en cuenta a la hora de valorar resistencias y diseñar estrategias compatibles con los distintos intereses que genera la cuestión.

\section{LÍNEAS DE ACTUACIÓN PARA DEFINIR UNA POLÍTICA SOBRE EL ALCOHOL}

Distintas actuaciones han sido propuestas en un intento por constituir una correcta política de actuación para reducir el abuso de alcohol y sus consecuencias. Cabe destacar, tanto por su elaboración como por su aplicabilidad en nuestro entorno, la Carta Europea sobre el Alcohol, aprobada por la Conferencia Europea sobre Salud, Sociedad y Alcohol, celebrada en 1995 en París. De igual manera, el Plan Europeo de Actuación sobre Alcohol 2000-2005, ofrece directrices comunes a todos los países europeos.

Por su extensión, obviamos reproducir los objetivos y acciones propuestos en el Plan
Europeo, centrándonos en aquellas más comunes que, por haber demostrado su efectividad deben constituir un conjunto mínimo de acciones. En este sentido, una política global contra el abuso de alcohol debería comprender intervenciones dirigidas a:

a) Disminuir la accesibilidad y disponibilidad de bebidas alcohólicas.

b) Actuar sobre la información y concienciación social, incluyendo las acciones sobre la publicidad de bebidas alcohólicas.

c) Reducir los daños asociados por consumo en determinadas situaciones $y$, en especial la violencia y la accidentabilidad de tráfico y laboral.

d) Asegurar una asistencia sanitaria basada en criterios de evidencia científica.

\section{Disminuir la accesibilidad y disponibilidad de bebidas alcohólicas.}

Un amplio abanico de actuaciones se han mostrado especialmente útiles para conseguir una disminución en la accesibilidad y disponibilidad de bebidas alcohólicas. Entre ellas destaca el incremento de los precios, las limitaciones en los horarios, zonas y días de venta, la edad mínima de consumo y, finalmente, la responsabilidad del personal implicado en su venta. Obviaremos, no obstante, otras medidas, por su dudosa aplicabilidad en nuestro país, tales como la prohibición, el racionamiento o el monopolio de la producción y venta.

Con relación a los precios de las bebidas alcohólicas, se ha constatado la evidencia de que un aumento de éstos determina un descenso en el consumo de alcohol ${ }^{11}$. Este descenso suele ser más acusado en grandes bebedores ${ }^{12}$ y mujeres ${ }^{13}$. Entre los jóvenes también parecen ser efectivas este tipo de medidas, tanto para disminuir el consumo como las embriagueces ${ }^{14}$. Como norma general, Grifith Edwards ${ }^{9}$ considera que el incremento de un $10 \%$ en el precio de las bebidas alcohólicas generan un descenso del $5 \%$ en 
el consumo de cerveza, $7.5 \%$ en el de vinos y $10 \%$ en el de licores.

Las limitaciones del consumo respecto a zonas, número de establecimientos, horarios y días han mostrado también resultados positivos. El incremento del número de puntos de dispensación, de los horarios y de los días en que se venden bebidas alcohólicas conlleva un aumento en el consumo per cápita de la población ${ }^{5}$. Con relación a la limitación del consumo en determinadas zonas (por ejemplo, en vía pública) nuestra experiencia apunta hacia un descenso en la prevalencia de abuso de alcohol, especialmente entre los grupos etarios más jóvenes. Así ha sido evidenciado en la Comunidad Valenciana, entre 1996 y 1998, con relación a la puesta en práctica de esta limitación de consumo ${ }^{15,16}$.

Finalmente, destaca la especial importancia que adquiere el límite de edad para el consumo de bebidas alcohólicas. El Plan Europeo de Actuación sobre Alcohol 2000-2005 aconseja establecer este límite en 18 años, tendencia que empieza a ser usual en toda Europa.

\section{Limitar la publicidad e incrementar la información y la concienciación social.}

Este tipo de medidas no solo incluye las clásicamente entendidas como de tipo preventivo (escolar, comunitario o laboral), sino las limitaciones de la publicidad y el correcto uso de ésta. Especial interés muestran las limitaciones en la publicidad, bien en el contenido de sus mensajes, bien en los horarios o medios de difusión. Edwards ${ }^{9}$ señala que los países con prohibiciones en la publicidad de licores presentan un consumo per cápita inferior en un $16 \%$ a aquellos que no disponen de estas limitaciones. A su vez, los accidentes de tráfico descienden un $23 \%$ cuando existen prohibiciones tanto en la publicidad de licores como de vino y cerveza.

Obviamente, el equilibrio necesario entre el comercio de bebidas alcohólicas y el daño generado por su abuso imposibilita optar por una completa prohibición. En su lugar, deben defenderse las limitaciones de horarios en los medios de radiodifusión, la distancia de la publicidad respecto a centros escolares, o su prohibición en determinados locales públicos. La inclusión de mensajes de advertencia fue iniciada en la Comunidad Valenciana en 1998, optando por su aparición en la publicidad y no en el etiquetado de las propias bebidas. Al margen de su posible -aunque discutida- utilidad para disminuir el consumo crónico de alcohol, los mensajes de advertencia permiten incrementar especialmente la percepción del riesgo ante el consumo en determinadas situaciones (por ejemplo, en la conducción o el embarazo), como han señalado distintos autores ${ }^{5}$.

Las medidas informativas de carácter más específico, como los programas preventivos a nivel escolar o laboral, deben constituir otro de los pilares básicos de este tipo de acciones. Si bien no parece existir una evidencia generalizada de la efectividad de las campañas y programas preventivos, es indudable su valor como instrumento de concienciación social. Tal vez deban realizarse dos advertencias al respecto. Por una parte, y como indica el Plan Europeo 2000-2005, estos programas deben tener como objetivo no solo la información sobre sustancias sino, muy especialmente, el aprendizaje de habilidades sociales como medio de afrontamiento ante las situaciones de consumo. Por otra, la necesidad de abandonar el concepto de "programa piloto" en favor de la generalización de estos programas a la absoluta totalidad de los centros escolares -cuando menos, de educación primaria y secundaria-, dentro del marco genérico de la Educación para la Salud.

\section{Reducir los daños asociados por consumo en determinadas situaciones.}

Como se observó en la Gráfica 1, no parece existir una relación directa entre la mortalidad por actos violentos, o los accidentes de tráfico, y el consumo per cápita en España. Esta ausencia de linealidad queda explicada por el hecho de que ambas causas de muerte se encuentran más relacionadas con el 
consumo agudo o explosivo de alcohol, que con un consumo crónico.

La intervención en este ámbito debe sustentarse, según se expone en el Plan Europeo 2000-2005, en la implementación de medidas disuasorias y restrictivas con relación a la alcoholemia máxima permitida para conducir vehículos (aconsejada en $0.5 \mathrm{mg} \%$ o inferior) así como en programas de tratamiento para conductores sancionados. Si bien la parte punitiva de esta estrategia suele ser cumplida con mayor o menor éxito, no cabe duda de la necesidad de extender, como práctica habitual, el desarrollo de programas obligatorios de tratamiento o consejo para conductores sancionados.

En el mismo sentido, la limitación de la venta y consumo de alcohol en lugares públicos masificados (en especial, acontecimientos deportivos y musicales) favorece un llamativo descenso de la violencia asociada. Igualmente, la detección precoz e intervención en casos de violencia doméstica asociada se entiende como acción cuasi obligatoria, habida cuenta de la relación existente con el abuso de alcohol, cifrada por distintos autores en un $40 \%$ de lo casos.

\section{Asegurar una asistencia sanitaria basada en criterios de evidencia científica.}

Los sistemas sanitarios públicos quedan obligados a ofrecer un tratamiento que, según se expone en el Plan Europeo, debe ser comprensivo, coordinado con otros recursos sociales, judiciales y de autoayuda, basados en la evidencia científica y suficientemente dotados a nivel económico. En otros términos, alcanzar la igualdad respecto al tratamiento de otras patologías debe ser el objetivo básico.

La asistencia sanitaria debe estructurarse, cuando menos, en dos niveles. Por una parte, la atención a nivel primario, de aquellos bebedores de riesgo, en los que sean útiles las intervenciones breves para disminuir o cesar el consumo. Este tipo de intervenciones puede llegar a disminuir un $23 \%$ el consumo de alcohol en este colectivo ${ }^{17}$. La capacitación de profesionales en los niveles primarios de la sanidad y los servicios sociales permitirá una importante reducción del daño asociado al consumo agudo, a la vez que favorecerá la motivación para el tratamiento en los casos de consumo crónico.

En un segundo nivel, dirigido específicamente al tratamiento de los casos con diagnóstico de dependencia o abuso, entendemos aconsejable la creación de unidades específicas integradas en el sistema sanitario público. Este modelo, desarrollado en la Comunidad Valenciana desde 1998, ha favorecido un importante incremento del volumen de pacientes que inician tratamiento, en un contexto normalizador de incalculable valor para una mejor evolución de los pacientes $^{18}$. En España, los objetivos planteados en la Estrategia Nacional sobre Drogas 20002008 incluyen un modelo similar al propuesto, claramente diferenciado de modelos basados en la beneficencia o en la atención prestada por entidades y administraciones sin competencias en materia sanitaria.

Como conclusión, tal vez sea conveniente recordar que España ocupa el décimo lugar, sobre un total de 15 países europeos, en el cumplimiento de las políticas de control sobre el alcohol, según el European Comparative Alcohol Study ${ }^{19}$. Una posición que debe ser comparada con la última posición ocupada hasta hace escasos años y que viene a demostrar el esfuerzo realizado en este sentido. Con todo, aún parece preciso insistir en algunas líneas de acción que como la homogeneidad de legislación autonómica y muy especialmente, el control sobre la publicidad y las tasas repercutidas sobre el alcohol - unas de las más bajas de Europa-, podrían situar a nuestro país en un lugar relevante entre las naciones que más están actuando en esta materia.

\section{BIBLIOGRAFÍA}

(*1) OBSERVATORIO ESPAÑOL SOBRE DROGAS. Informe $\mathbf{N}^{\circ}$ 4. Marzo 2001. Ministerio del Inte- 
rior. Delegación del Gobierno para el Plan Nacional sobre Drogas. Madrid, 2001.

(**2) PORTELLA, E. ET AL. El alcohol y su abuso: impacto socioeconómico. Ed. Médica Panamericana. Madrid, 1998.

(3) ROBLEDO DE DIOS, T. Alcohol as important health determinant in Spain and EU. WHO European Ministerial Conference on Young People and Alcohol. Stockholm, 2001.

(*4) MINISTERIO DEL INTERIOR. Estrategia Nacional sobre Drogas 2000-2008. Delegación del Gobierno para el Plan Nacional sobre Drogas. Madrid, 2000.

(**5) EDWARDS, G. ET AL. Alcohol y Salud Pública. Prous Science. Barcelona, 1997.

(6) PATAKI, G.E.; MILLER, J.S. The costs and consequences of addiction and the benefits of prevention and treatment. New York State Office of Alcoholism and Substance Abuse. New York, 1998.

(7) SINGLE, E. ET AL. The costs of substance abuse in Canada. Canadian Centre on Substance Abuse. Toronto, 199.

(**8) WORLD HEALTH ORGANIZATION. Global Status Report on Alcohol. Substance Abuse Department. Geneva, 1999.

(**9) REHN, N.; ROOM, R.; EDWARDS, G. Alcohol in the European Region: consumption, harm and policies. World Health Organization. Regional Office for Europe, 2001.

(**10) WORLD HEALTH ORGANIZATION. International Guide for monitoring alcohol consumption and related harm. Department on Mental Health and Substance Dependence. Geneva, 2000.
(11) RAISTRICK, D.; HODGSON, R.; RITSON, B. Tackling alcohol together. Free Association Books. London, 1999.

(12) SUTTON, M. y GODFREY, C. A grouped data regression approach to estimating economic and social influences on individual drinking behaviour. Health Economics, 1995, 4: 237-247.

(13) KENKEL, D.S. Drinking, driving and deterrence: the social costs of alternative policies. Journal of Law and Economics, 1993, 36: 877-914.

(*14) GODFREY, C. Young people, economics, alcohol and cost effective policies. WHO European Ministerial Conference on Young People and Alcohol. Stockholm, 2001.

(15) PÉREZ, B.; HERNÁNDEZ, J.J.; NAVARRO, J. EI consumo de drogas y factores asociados en la Comunidad Valenciana. Generalitat Valenciana-Fundación de Ayuda contra la Drogadicción. Madrid, 1997.

(16) NAVARRO, J.; MEJÍAS, E.; GÓMEZ, E. El consumo de drogas y factores asociados en la Comunidad Valenciana (II). Generalitat Valenciana-Fundación de Ayuda contra la Drogadicción. Madrid, 1999.

(*17) HEATHER, N. Treatment approaches to alcohol problems. World Health Organization. WHO Regional Publications European Series, $n^{\circ}$ 65. Copenhagen, 1995 (p. 63).

(18) GENERALITAT VALENCIANA. Sistema de Evaluación de la Calidad Asistencial en Drogodependencias (1998-2000). Generalitat Valenciana. Conselleria de Bienestar Social. Valencia, 2001. (En prensa).

(19) OSTERBERG, E. Trends in alcohol control policy in countries of the European Union, 1950-2000. 